\title{
Di-boson production measurements with the ATLAS detector
}

\author{
L.Chevalier* on behalf of the Atlas Collaboration \\ CEA-Saclay, F-91191 Gif-sur-Yvette, France
}

E-mail: laurent.chevalier@cea.fr

\begin{abstract}
This talk covers the results of the measurements of diboson production cross sections in protonproton interactions at $\sqrt{s}=7 \mathrm{TeV}$ in 2011 and at $\sqrt{s}=8 \mathrm{TeV}$ in 2012, performed by the ATLAS experiment. The cross sections are measured in phase space regions defined by the decay kinematics, the detector acceptance and then extrapolated to the full phase space. Cross sections for $\mathrm{VV}(\mathrm{V}=\mathrm{W}, \mathrm{Z}, \gamma)$ production in the leptonic or semileptonic channels are compared to $(\mathrm{N}) \mathrm{NLO}$ predictions of the Standard Model. Kinematic spectra are used to place constraints on anomalous triple-gauge-boson couplings. All cross sections are in agreement with the Standard Model predictions. No experimental evidence is found of anomalous triple-gauge-boson couplings.
\end{abstract}

The European Physical Society Conference on High Energy Physics

22-29 July 2015

Vienna, Austria

${ }^{*}$ Speaker. 


\section{Introduction}

All LHC [1] processes measured by ATLAS experiment [2] lead to a good or a very good agreement with the theory. The majority of the results are within $2 \sigma$ of the predictions, over 14 orders of magnitude, see figure 1. Higgs boson observation is the main electroweak result but the observation of WW scattering is also a very important for the Standard Model (SM) consistency. Although all these results are conform to the SM within errors, some open questions remain; Dark matter, mass hierarchy, neutrinos and many others. A way to test deeply the SM and maybe access to new physics contributions is to perform precise measurements on dibosons processes. Main results from the dibosons area are presented below.

\subsection{ATLAS Detector}

The ATLAS detector is a multi-purpose particle physics detector with cylindrical geometry and approximately forward-backward symmetric. The inner tracking detector (ID) with a pseudorapidity coverage of $|\eta|<2.5$, consists of a silicon pixel detector, a silicon micro-strip detector, and a transition radiation tracker. The ID is surrounded by a thin superconducting solenoid providing a 2 Tesla axial magnetic field. A high-granularity lead/liquid-argon sampling calorimeter (LAr) measures the energy and the position of electromagnetic showers within $|\eta|<3$.2. LAr sampling calorimeters are also used to measure hadronic showers in the end-cap $(1.5<|\eta|<3.2)$ and forward $(3.1<|\eta|<4.9)$ regions, while an iron/scintillator tile calorimeter measures hadronic showers in the central region $(|\eta|<1.7)$. The muon spectrometer (MS) surrounds the calorimeters and consists of three large superconducting air-core toroid magnets ( 0.5 Tesla in average in the barrel), each with eight coils, a system of precision tracking chambers $(|\eta|<2.7)$, and fast tracking chambers for triggering $(|\eta|<2.4)$, see figure 2 .

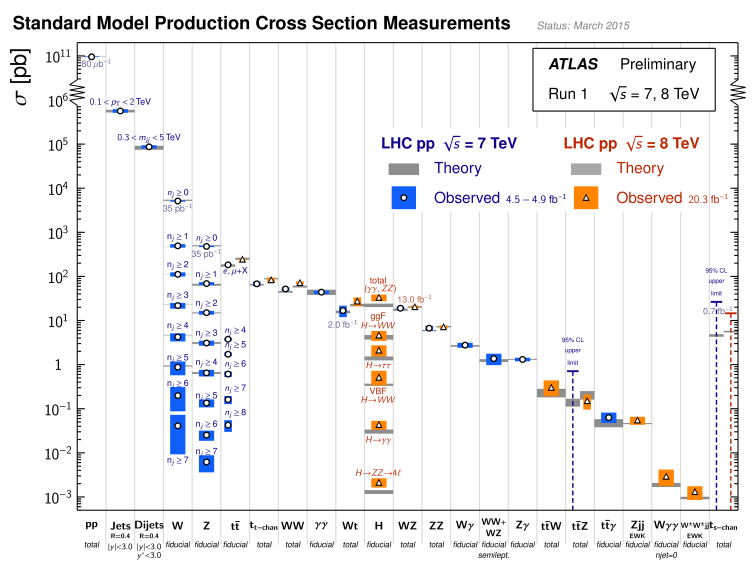

Figure 1: Summary of several Standard Model total and fiducial production cross section measurements, compared to the corresponding theoretical expectations [3, 4].

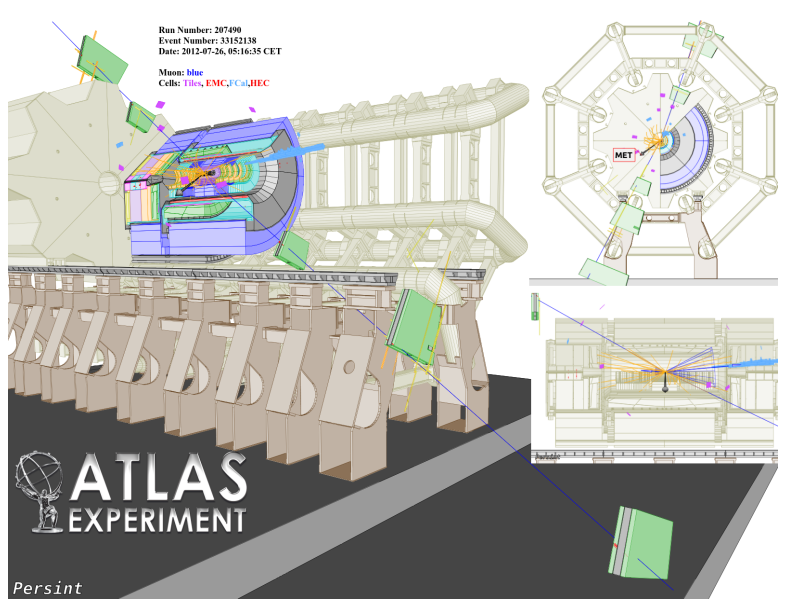

Figure 2: Event display of a $W W$ candidate with two leptons with the same sign and two jets. Subdetectors are partially drawn for clarity. 


\subsection{Triple Gauge Couplings in the Standard Model}

The non-abelian structure of $\mathrm{SU}(2) \otimes \mathrm{U}(1)$ in the SM predicts the existence, at tree level, of the charged Triple Gauge Couplings (TGC) and Quartic Gauge Couplings (QGC), while vertices with all bosons neutral are forbidden. The TGC vertex is completely fixed by the electroweak gauge structure and so a precise measurement of this vertex, through the analysis of diboson production, allows to test the high energy behaviour of electroweak interactions. The strength of the couplings is set by the universal gauge couplings of the $\mathrm{SU}(2)$ local gauge symmetry. Any deviation from gauge constraints can cause modifications of the production cross section at high diboson invariant mass. We can add to the SM Lagrangian, new TGC's by just requiring the Lorentz invariance with the conservation of $U(1)$ for neutral couplings $[6,7]$ or without this $U(1)$ conservation $[8,9]$. The presence of these new couplings or anomalous triple (and quartic) gauge boson couplings (aTGC) can be measured and would be a signature of new physics.

\section{2. $\mathbf{W} \gamma$ and $\mathrm{Z} \gamma$ cross section at $7 \mathrm{TeV}$}

Two sets of cross section measurements are performed, inclusive and exclusive, with final states with leptons and photons. The inclusive cross section measurement refers to production with no restriction on the number of jets produced. The exclusive measurement refers to events with no central jets with transverse energy ${ }^{1} \mathrm{E}_{\mathrm{T}}^{\gamma}>30 \mathrm{GeV}$. Jets are reconstructed from the energy observed in the calorimeter cells using the anti-kt jet clustering algorithm [3]. The main background processes are $Z+$ jets, $W+$ jets and $\gamma+$ jets but there are also contributions from $t \bar{t}$ and $W W$ productions. The systematic uncertainties on the cross sections are dominated by uncertainties on the photon identification, background subtraction and jet energy scale.
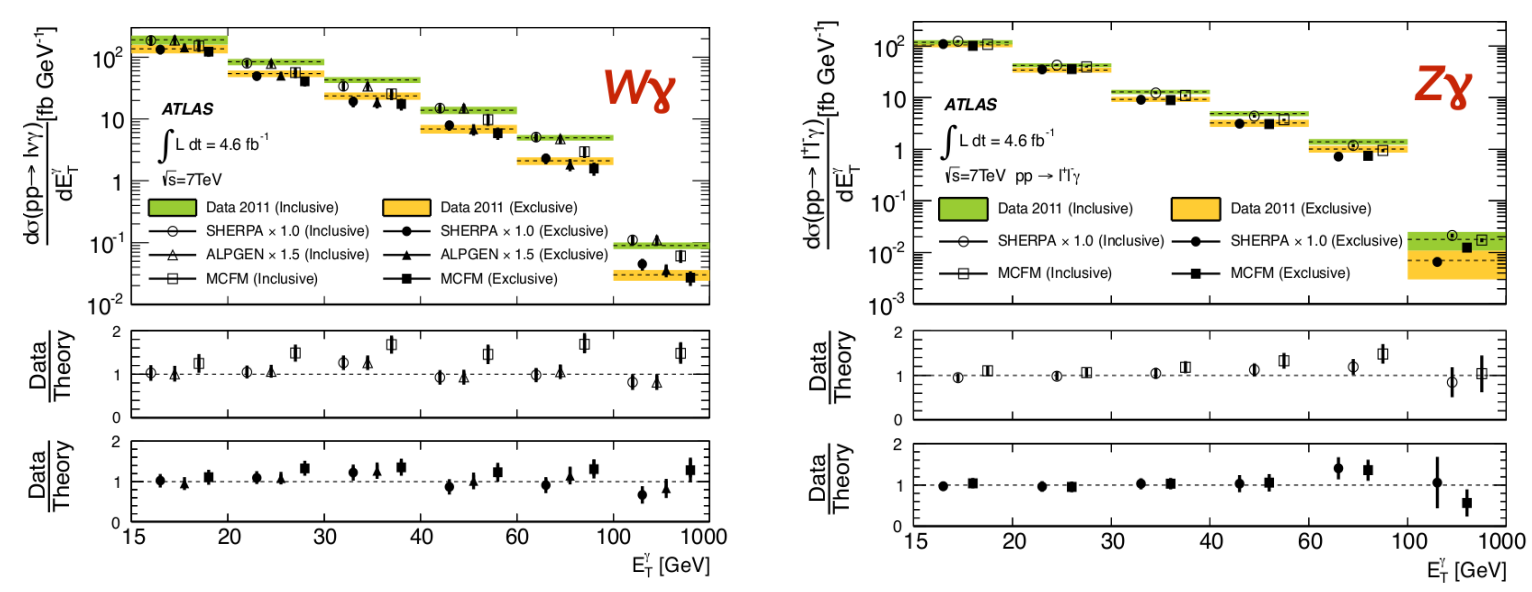

Figure 3: Measured differential $\left(\mathrm{E}_{\mathrm{T}}^{\gamma}\right)$ cross sections as the function of transverse energy for the $\mathrm{pp} \rightarrow l v \gamma$ (left) and $\mathrm{pp} \rightarrow l^{+} l^{-} \gamma$ (right) processes, using the combined electron and muon measurements for the inclusive $(\mathrm{Njet} \geq 0)$ and exclusive $(\mathrm{Njet}=0)$ fiducial regions. The lower plots show the ratio of the data to the predictions for different generators. The Monte Carlo uncertainties are shown only in the ratio plots [4].

In figure 3 the measured $\mathrm{E}_{\mathrm{T}}^{\gamma}$ differential cross sections for $\mathrm{pp} \rightarrow W \gamma \rightarrow l v \gamma$ (left) and $\mathrm{pp} \rightarrow Z \gamma \rightarrow$ $l^{+} l^{-} \gamma$ (right) are shown for both the inclusive and exclusive measurements. Alpgen and Sherpa

\footnotetext{
${ }^{1}$ remark:a better wording should be:missing transverse momentum
} 
generators describe the $W \gamma$ and $Z \gamma$ data distributions quite well for both topologies. The inclusive $W \gamma$ measurement is underestimated by the MCFM generator. This is attributed to missing higherorder QCD contributions beyond the available NLO prediction .For the $Z \gamma$, the agreement is quite good. A new fully differential computation of radiative corrections at next-to-next-to-leading order (NNLO) in QCD perturbation theory has become available since these analyses were published and can be found in references[5]. Corrections are sizeable for the $W \gamma$ case, which can reach up to $\sim 20 \%$ with respect to NLO calculations. Smaller corrections for $Z \gamma$ are reported. In figure 4, we see that the data agree with the NLO and NNLO theoretical predictions within the uncertainties, and that the NNLO corrections slightly improve this agreement.

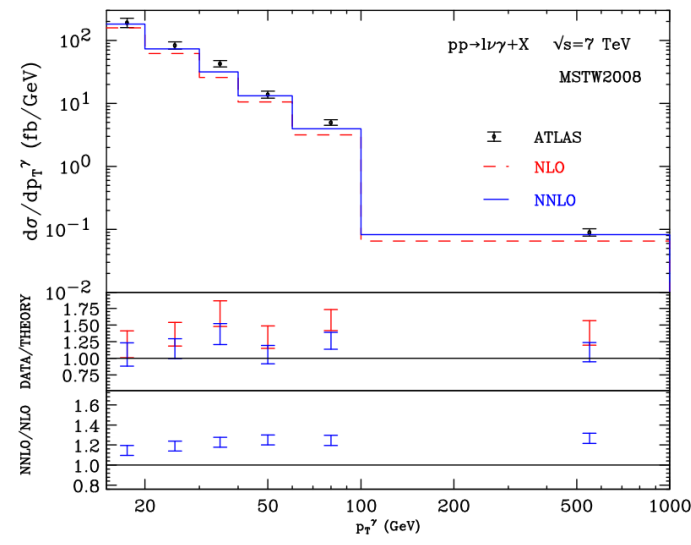

Figure 4: The transverse energy distribution of the photon in $W \gamma$ production, computed at NLO (dashes) and NNLO (solid) compared to the ATLAS data. The middle panel shows the ratio data/theory. The lower plot shows the ratio NNLO/NLO [5].

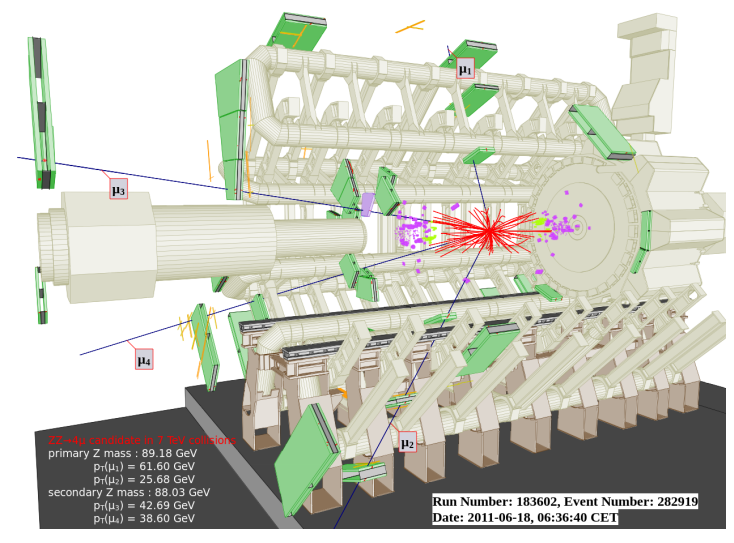

Figure 5: A typical example of $\mathrm{ZZ}$ event in $4 \mu$.

\section{3. $\mathrm{WZ}$ and $\mathrm{ZZ}$ cross section at $7 \& 8 \mathrm{TeV}$}

The analyses of $W Z$ boson pairs are performed in $3 l v$ final states. The $\mathrm{Z}$ boson pair production is performed in the $4 l$, see event display example in figure 5, and in $2 l 2 v^{2}$ final states $[11,13$, $12,14]$. The main sources of background to the $W Z \rightarrow 3 l v$ final state come from $Z+j e t s$ and $t \bar{t}$ events, where the two leptons from the vector boson decays are accompanied by a jet which is misidentified as a lepton. These backgrounds are estimated from data-driven techniques. The $Z Z$ event contributes to the background too; one of the leptons falls outside the acceptance of the detector and thus creates $E_{T}^{\text {miss }}$. This source is estimated from MC. For the $Z Z$ final state, the four leptons final state is a clean signature with small background contributions. These mainly come from $\mathrm{Z}+\mathrm{jets}$ and $\mathrm{t} \overline{\mathrm{t}}$ processes where the jets are misidentified as leptons and are estimated with data-driven methods. The background is more significant in the $2 l 2 v$ final state and is a mixture of diboson, $t \bar{t}$ and Drell-Yan events. The dominant uncertainty in the $W Z$ cross section measurement is the systematic uncertainty in the data driven background estimation. The $Z Z$ measurement is

\footnotetext{
${ }^{2}$ only at $7 \mathrm{TeV}$
} 
dominated by statistical uncertainties while the systematics are dominated by lepton identification and momentum resolution. The results on the total production cross-section for both measurements at 7 and $8 \mathrm{TeV}$ are presented in figure 6 and compared to SM predictions. Overall good agreement with the predictions from the SM is found.
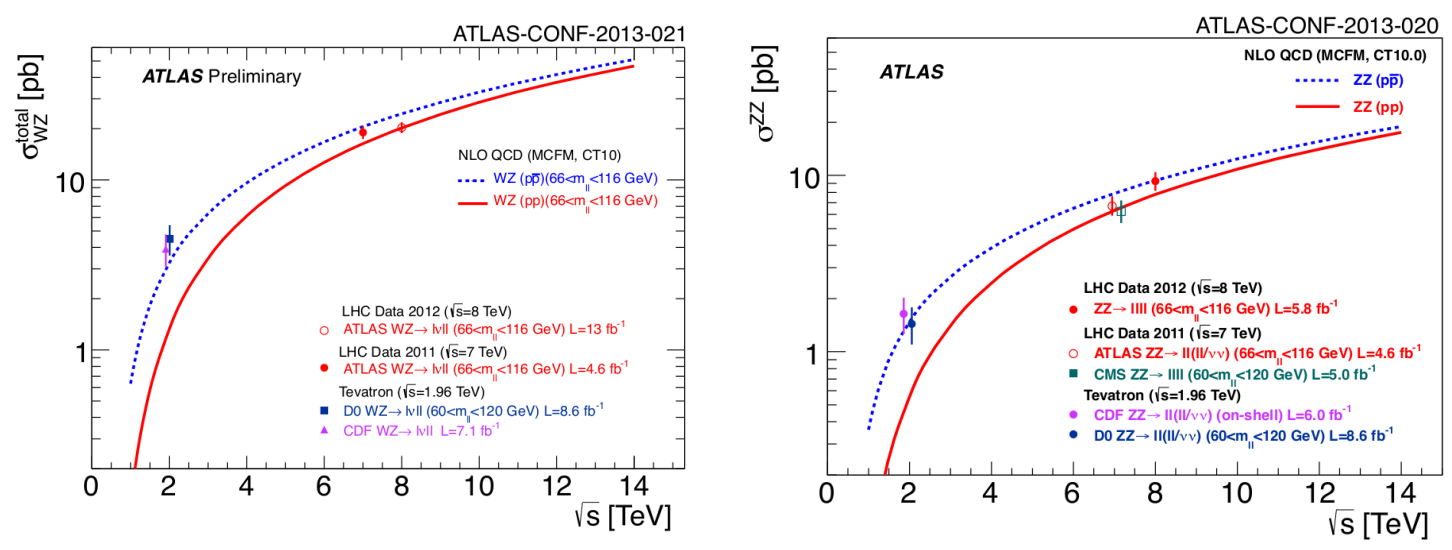

Figure 6: Measurements and theoretical predictions of the total $W Z$ (left) and $Z Z$ (right) production cross section as a function of centre of mass energy $\sqrt{s}[13,14]$. Experimental measurements from CDF [16], D0 [15] in p $\bar{p}$ collisions, for the ZZ channel, the CMS [17] and for ATLAS in pp collisions are shown. The blue dashed line shows the theoretical prediction for the $W Z / Z Z$ production cross section in p $\bar{p}$ collisions. The solid red line shows the theoretical prediction for the $W Z / Z Z$ production cross section in pp collisions.

\section{4. $\mathrm{WW}+\mathrm{WZ}$ in semi-leptonic mode at $7 \mathrm{TeV}$}

The candidates $W V(V=W$ or $Z) \rightarrow l v j j$ events are required to contain exactly one lepton, large missing transverse momentum and exactly two jets. The selected events are accepted if they pass a set of kinematic cuts chosen to enhance the signal-to-background ratio. The $W W+W Z$ signal yield is obtained by performing a binned maximum-likelihood fit to the dijet mass $\left(m_{j j}\right)$ distribution using templates based on MC. The fit is performed on events in a $m_{j j}$ range much larger than the range where the signal peaks, allowing the nearly signal free dijet regions to constrain the rate of the $W+$ jets events, which are the largest background. Other backgrounds are the multi-jet, top and diboson events. Multi-jet background, like the dominant $W+$ jets, is estimated with data-driven techniques, while the other two are estimated from MC. The figure 7 shows the dijet invariant mass distribution after the likelihood fit for the combined electron and muon final states. The normalisations and shapes of the histograms are obtained from the best fit to the data, after being allowed to vary within their systematic uncertainties. Good overall agreement is seen between the data and the total fit result as shown in the lower panel display which plots the ratio between the data and the total fit result, including both signal and backgrounds. The agreement between data and the fit is within $2 \%$. The dijet mass distribution of the background-subtracted data for the sum of the electron and muon channels is shown on the figure 8 . The $W / Z \rightarrow \mathrm{jj}$ resonance is observed and in good agreement with the expectation from the SM. 

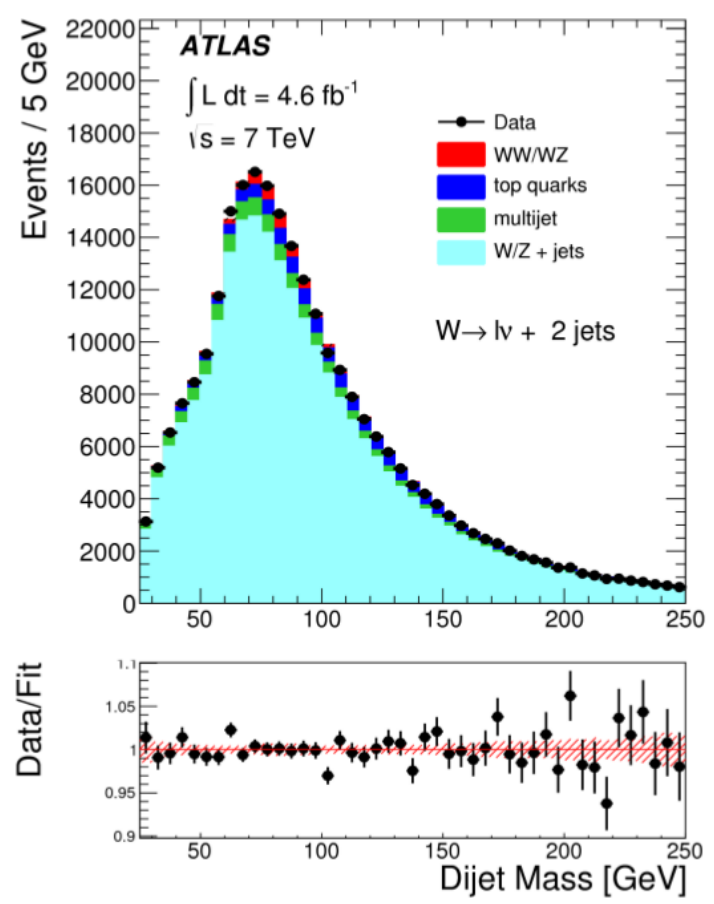

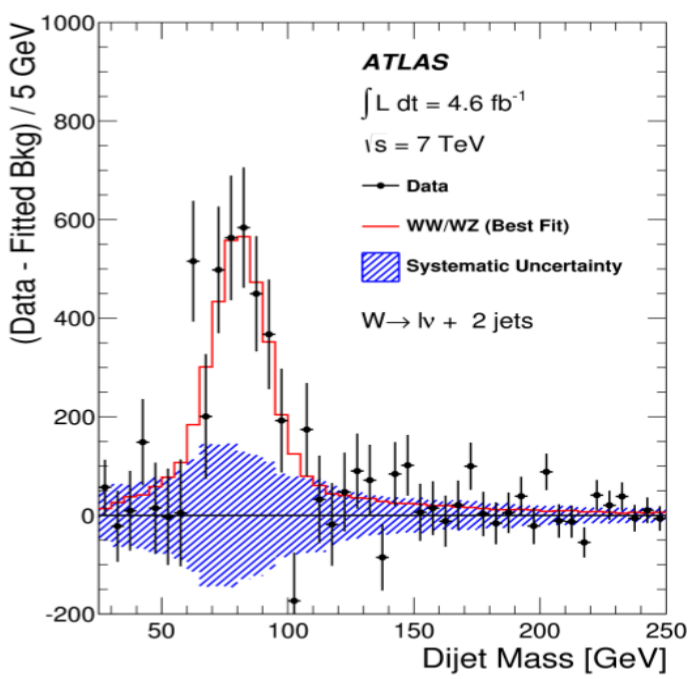

Figure 8: Dijet invariant mass distribution of the background-subtracted data for the sum of the electron and muon channels. The error bars represent the statistical error on the data[18].

Figure 7: Dijet invariant mass distributions after the likelihood fit for the combined electron and muon topology final states. The lower panel displays the ratio between the data and the total fit result, including both signal and backgrounds [18].

\section{WW cross section at $8 \mathrm{TeV}$}

The $W W \rightarrow l v l v$ signal is measured in final states with two oppositely charged isolated leptons and large missing transverse energy [?, 19]. Candidate $W W$ events are required to have no jets reconstructed in the final state because the background dominates at higher jet multiplicities. This comparison between the measured combined $W W$ production cross section (red+blue line with gray shading) and the predictions using different PDF sets is shown in figure 9 . The jet multiplicity distributions is shown for $e \mu$ events, data and Monte Carlo simulation, before the jet-veto requirement is applied. Processes that can mimic the $l l+E_{T}^{\text {miss }}$ signal with no reconstructed jets are top-quark production, when then event contains jets falling outside the acceptance and thus passing the jet veto requirement, $W+$ jets when a jet fakes a lepton and Drell-Yan with mis-measured jets and diboson channels. The driving uncertainty on the measurement is the systematic uncertainties associated with the jet veto requirement and the estimation of backgrounds from "fake" leptons using data driven methods. The figure 10 summarizes the results of the $W W$ analysis. The cross sections measured in the individual channels as well as the combined cross section are shown and compared to the SM prediction, which is obtained using the CT10 PDF set. The yellow and green shaded bands represent the PDF and total theoretical uncertainties respectively. The filled symbols show the measured total cross section with the statistical and total uncertainty. The individual channels are compatible within their uncertainties, however all measurements lie above the SM 
prediction. Since this result was presented there has been an effort to improve the theoretical calculations by computing NNLO corrections which can account a part of the difference, while other calculations based on re-summation of large logarithms that arise from a jet-veto condition, provide further corrections. These results are reported in references [21, 22, 23].

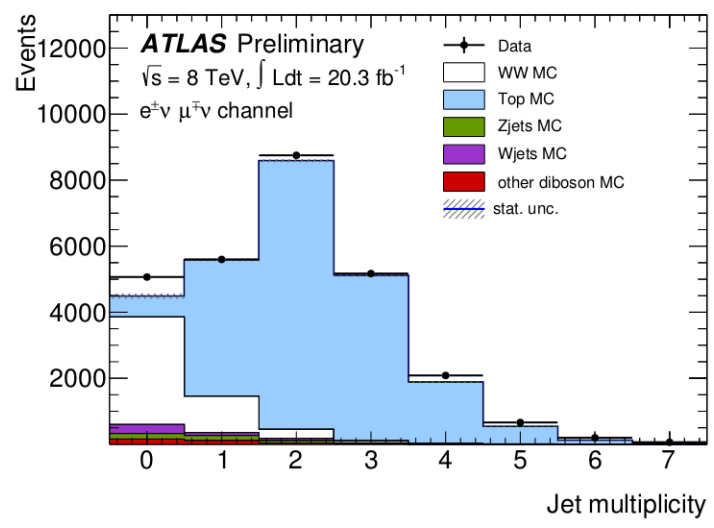

Figure 9: Jet multiplicity distribution for $e \mu$ events. The points represent data and the stacked histograms are the MC predictions. Only statistical uncertainties are shown [20].

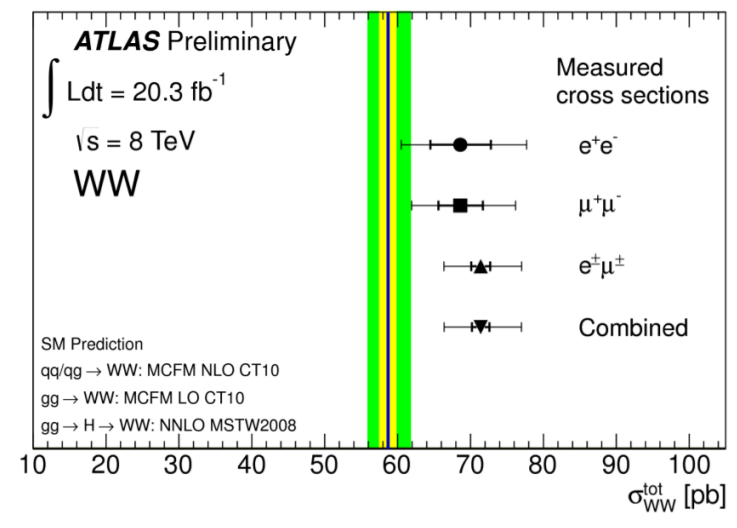

Figure 10: Comparison between predicted WW production cross section at $\sqrt{s}=8 \mathrm{TeV}$ using CT10PDF and the measured cross section. The yellow and green shaded bands represent the PDF and total theoretical uncertainties respectively. The filled symbols show the measured total cross section with the statistical and total uncertainty [20].

\section{Anomalous TGC (aTGC)}

The figure 11 shows a summary of the limits set on the different aTGC parameters along with comparisons to other experiments. No deviation from the expected SM values is observed in any channel.

\section{Summary of cross section}

Measurements of the production cross sections for $7 \& 8 \mathrm{TeV}$ have been performed by ATLAS and for all processes listed in the Table 1 with the theoretical predictions.

\section{Conclusion}

The ATLAS Run-1 allowed to explore diboson production processes, all results are perfectly consistent with the SM. However diboson measurements are limited by the statistic. The RUN-2 energy in the centre of mass and the foreseen luminosity should allow to access to the triboson processes. 

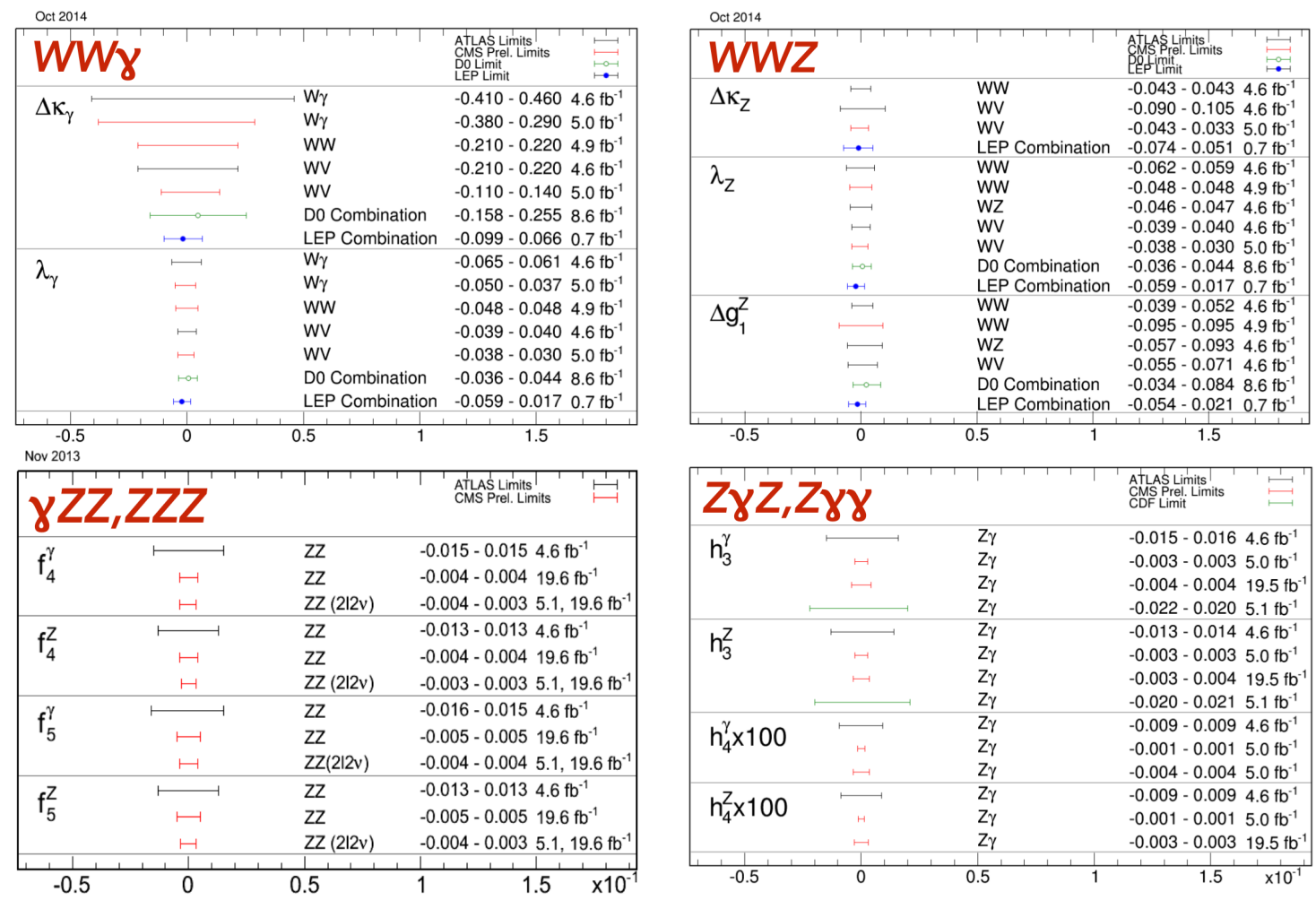

Figure 11: Top plots: Comparison of results from ATLAS, CMS, CDF, D0 and LEP experiments on limits on charged anomalous triple gauge couplings at $95 \% \mathrm{CL}$. Bottom plots: Comparison of results from ATLAS, CMS, CDF, D0 and LEP experiments on limits on neutral anomalous triple gauge couplings at 95\%CL [24].

\begin{tabular}{|l|c|c|c|c|c|c|c|c||}
\hline$\sqrt{s}$ & $\int L \mathrm{~d} t\left(\mathrm{fb}^{-1}\right)$ & Process & data $(\mathrm{pb})$ & stat. & syst. & lumi. & theory & err. \\
\hline $7 \mathrm{TeV}$ & 4.6 & $\mathbf{W} \gamma$ & 2.77 & ${ }_{-0.03}^{+0.03}$ & ${ }_{-0.33}^{+0.33}$ & ${ }_{-0.14}^{+0.14}$ & 2.456 & ${ }_{-0.006}^{+0.006}$ \\
$7 \mathrm{TeV}$ & 4.6 & $\mathbf{Z} \gamma$ & 1.31 & ${ }_{-0.02}^{+0.02}$ & ${ }_{-0.11}^{+0.11}$ & ${ }_{-0.05}^{+0.05}$ & 1.305 & ${ }_{-0.003}^{+0.003}$ \\
\hline \hline $7 \mathrm{TeV}$ & 4.6 & $\mathbf{W Z}$ & 19.0 & ${ }_{-1.3}^{+1.4}$ & ${ }_{-0.9}^{+0.9}$ & ${ }_{-0.4}^{+0.4}$ & 17.6 & ${ }_{-1.0}^{+1.1}$ \\
$8 \mathrm{TeV}$ & 13.0 & $\mathbf{W Z}$ & 20.3 & ${ }_{-0.7}^{+0.8}$ & ${ }_{-1.1}^{+1.2}$ & ${ }_{-0.8}^{+0.7}$ & 20.3 & ${ }_{-0.8}^{+0.8}$ \\
\hline \hline $7 \mathrm{TeV}$ & 4.6 & $\mathbf{Z Z}$ & 6.7 & ${ }_{-0.7}^{+0.7}$ & ${ }_{-0.3}^{+0.4}$ & ${ }_{-0.3}^{+0.3}$ & 5.89 & ${ }_{-0.18}^{+0.22}$ \\
$8 \mathrm{TeV}$ & 20.3 & $\mathbf{Z Z}$ & 7.1 & ${ }_{-0.4}^{+0.5}$ & ${ }_{-0.3}^{+0.3}$ & ${ }_{-0.2}^{+0.2}$ & 7.2 & ${ }_{-0.2}^{+0.3}$ \\
\hline \hline $7 \mathrm{TeV}$ & 4.6 & $\mathbf{W W + W Z}$ & 68.0 & ${ }_{-7.0}^{+7.0}$ & ${ }_{-19}^{+19}$ & & 61.1 & ${ }_{-2.2}^{+2.2}$ \\
\hline \hline $7 \mathrm{TeV}$ & 4.6 & $\mathbf{W W}$ & 51.9 & ${ }_{-2.0}^{+2.0}$ & ${ }_{-3.9}^{+3.9}$ & ${ }_{-2 .}^{+2 .}$ & 44.7 & \\
$8 \mathrm{TeV}$ & 20.3 & $\mathbf{W W}$ & 71.4 & ${ }_{-1.2}^{+1.2}$ & ${ }_{-4.4}^{+5.0}$ & ${ }_{-2.1}^{+2.2}$ & 58.7 & \\
\hline
\end{tabular}

Table 1: Summary of production cross section of $W \gamma, Z \gamma($ atNNLO)and $W Z, Z Z, W W+W Z \& W W$ channels and comparison with theoretical calculations.

\section{References}

[1] L. Evans and P. Bryant (editors), JINST 3 (2008) S08001.

[2] ATLAS Collaboration, JINST 3 (2008) S08003.

[3] ATLAS Collaboration, PRD 87, 112003 (2013), http://arxiv.org/abs/1302.1283.

[4] ATLAS Collaboration, PRD 87, 112003 (2013), arXiv:1302.1283.

[5] M. Grazzini, http://arxiv.org/abs/1407.1618. 
[6] G.J.Gounaris et al. Phys. Rev. D 61, 0730132000.

[7] C.Degrande et al. Annals of Physics, Volume 335, August 2013, Pages 21-32, ISSN 0003-4916.

[8] K.Hagiwara et al. Nuclear Physics B, Volume 282, 1987, Pages 253-307, ISSN 0550-3213.

[9] C.Degrande, Journal of High Energy Physics 02-2014, 2014:101.

[10] ATLAS Collaboration, Phys. Rev. D 87, Erratum Phys. Rev. D 91, 119901 (2015).

[11] ATLAS Collaboration, Eur. Phys. J., C72 (2012) 2173.

[12] ATLAS Collaboration, JHEP, 03 (2013) 128.

[13] ATLAS Collaboration, ATLAS-CONF-2013-021, http://cdsweb.cern.ch/record/1525557.

[14] ATLAS Collaboration, ATLAS-CONF-2013-020, http://cdsweb.cern.ch/record/1460409.

[15] V. M. Abazov et al. (The DO Collaboration), Phys. Rev. D 85, 112005.

[16] T. Aaltonen et al. (The CDF Collaboration), Phys. Rev. Lett. 108, 101801.

[17] S. Chatrchyan et al. (The CMS Collaboration), JHEP 1301 (2013) 063.

[18] ATLAS Collaboration, 10.1007/JHEP01(2015)049.

[19] ATLAS Collaboration, Phys. Rev. D 87, 112001 (2013), arXiv:1210.2979.

[20] ATLAS Collaboration, ATLAS-CONF-2014-033, https://cdsweb.cern.ch/record/1728248.

[21] T.Gehrmann et. al, arXiv:1408.5243.

[22] P.Jaiswal et al. arXiv:1407.4537.

[23] P.Meade et al. arXiv:1407.4481.

[24] https://twiki.cern.ch/twiki/bin/view/CMSPublic/PhysicsResultsSMPaTGC. 